\title{
Nuevo análisis de "La tragedia de los comunes"
}

Miguel Esteban Cloquell ${ }^{*}$

Departamento de Ciencias División de Ciencias e Ingeniería Universidad de Quintana Roo

\section{Resumen}

El propósito del presente trabajo es analizar las implicaciones éticas del artículo de Garrett Hardin "La tragedia de los comunes" (1968). Tras una introducción, en el segundo apartado se formula el desafío ambiental lanzado por el autor, mostrando su dependencia de la teoría clásica o instrumental de la racionalidad, según la cual la dimensión axiológica queda excluida de la acción racional. En el tercero se examina críticamente la tesis de Hardin, quien propone considerar la noción ecológica de capacidad de carga como un concepto ético, desestimando su pretensión de derivar de ésta una ética antialtruista. En el cuarto se aborda el análisis del problema de Hardin por Elinor Ostrom, quien indica la presencia de nociones éticas de reciprocidad en las comunidades que han logrado minimizar los efectos ambientales pronosticados por el autor. En el quinto se equipara esquemáticamente la estructura de la tragedia de los comunes con la de la crisis del cambio climático, analizando las condiciones de cooperación necesarias para hacer frente a esta última y mostrando que, por muy avanzadas que estén nuestras soluciones tecnológicas, sin una ética de la ciudadanía la tragedia parece inevitable. Esta exigencia queda establecida en las conclusiones.

\section{Palabras Clave}

Hardin, capacidad de carga, ética ambiental, racionalidad axiológica, reciprocidad. 


\title{
New Analysis of "The Tragedy of the Commons"
}

\author{
Miguel Esteban Cloquell \\ Departamento de Ciencias \\ División de Ciencias e Ingeniería \\ Universidad de Quintana Roo
}

\begin{abstract}
This paper is an analysis of the ethical implications of Hardin's "The Tragedy of the Commons" (1968). After an Introduction, section two states Hardin's environmental challenge, showing how his formulation depends on the classic or instrumental theory of rationality, which excludes axiological considerations from rational action. Section three deals critically with Hardin's ethical concept of carrying capacity, ruling out his claim to an ethics against altruism. Section four examines Ostrom's view on the problem, which indicates the presence of ethical notions of reciprocity in those communities which reduce the environmental effects forecasted by Hardin. In section five common structure is identified between the tragedy of the commons and climate change crisis, analyzing the conditions of cooperation for facing it, and showing that, no matter how updated our technologies could be, environmental tragedy seems unavoidable in absence of an ethics of citizenship. This demand is stated as the last of the final remarks of the work.
\end{abstract}

\section{KEY WORDS}

Hardin, carrying capacity, environmental ethics, axiological rationality, reciprocity. 


\section{Introducción}

El artículo de Garrett Hardin "The Tragedy of the Commons” $(1968)^{\dagger}$ se ha convertido en referencia obligada en el debate relativo a la capacidad de carga del planeta para sustentar el crecimiento demográfico de las sociedades industriales contemporáneas, sobrepasada la cual la catástrofe ecológica resulta inevitable. Como Heidegger, Heilbroner y muchos otros habían hecho con anterioridad, Hardin advertía la inadecuación de las políticas sociales de las democracias desarrollistas y "humanitarias" (el término es de Hardin) para enfrentarse a la degradación ambiental inducida por la extensión de la tecnología como fenómeno planetario. El objetivo de este trabajo es analizar desde la perspectiva de la racionalidad ambiental los presupuestos conceptuales y empíricos de la TC y las consecuencias que Hardin extrae de ésta, revelando cómo los conceptos de bien común y reciprocidad resultantes de este análisis crítico son pertinentes para integrar una ética de la ciudadanía entre las disciplinas propias de la educación ambiental para la sustentabilidad. Ello requiere desestimar la ética antialtruista, situacionista o supervivencialista que Hardin deriva de la TC a partir del concepto de capacidad de carga. Será pues necesario mostrar que su formulación de la TC depende de la decisión de mantener sin revisar la teoría clásica o instrumental de la racionalidad, cuya exclusión de los aspectos axiológicos de la acción racional (Weber, 1981: 40) impide desde el principio la cooperación necesaria para hacer frente a la tragedia.

\section{Acción económica racional en recursos comunes}

Hardin formula la TC como un experimento mental. Imagínese un pastizal en el que puede pastar el ganado de todos los miembros de una comunidad de pastores. En este escenario, cada pastor intentará mantener en los recursos comunes tantas cabezas de ganado como le sea posible. "Como un ser racional, cada pastor busca maximizar su ganancia” (Hardin, 1968: 1244), pues el incremento de una cabeza de ganado trae al pastor los beneficios íntegros derivados de su venta (1), mientras que los costos debidos al sobrepastoreo producidos por ese incremento son compartidos por todos los pastores, por lo que, al resultar del cociente entre el total $n$

+ "La tragedia de los comunes”; en adelante TC. 
de pastores $(1 / \mathrm{n})$, serán necesariamente menores a 1. Siendo pues el beneficio (1) $n$ veces el costo $(1 / n)$, "el pastor racional concluye que la única decisión sensata para él es añadir otro animal a su rebaño" (Hardin, 1968: 1244). Pero ésta es la conclusión a la que llegan todos y cada uno de los pastores racionales que comparten el recurso común. Y ahí reside la tragedia. Cada agente individual está atado a una racionalidad que lo impulsa a incrementar su ganado ilimitadamente en un pastizal que resulta finito en cuanto recurso.

Conviene reparar en que, tal como Hardin plantea el problema, es el determinismo del resultado lo que constituye la tragedia. La conclusión bien pudo haber sido que, por sí sola, la racionalidad instrumental —entendida como la maximización de las relaciones de eficiencia entre medios y fines (o entre costos y beneficios) conduce inexorablemente al agotamiento de los recursos comunes. Con todo, el autor opta por considerar irrevisable esa racionalidad, y prefiere deducir de la TC una advertencia sobre las trágicas consecuencias ecológicas de los regímenes de propiedad comunal que privilegian la justicia distributiva. Entonces, llega al extremo de extraer el siguiente corolario: "La injusticia es preferible a la ruina de todos" (Hardin, 1968: 1244).

En realidad, su formulación originaria parecía sugerir lo contrario: la incompletitud de la racionalidad instrumental o técnica para hacer frente a problemas ambientales como los que plantea el libre uso de los recursos comunes. En este sentido, la TC es para él simplemente una instancia de lo que denomina "problemas sin solución puramente técnica”:

Una suposición implícita y casi universal de los análisis publicados en revistas científicas profesionales y de divulgación es que todos los problemas discutidos tienen una solución técnica. Una solución de este tipo puede definirse como aquella que sólo requiere un cambio en las técnicas de las ciencias naturales, demandando pocos o casi nulos cambios en relación con los valores humanos o en las ideas de moralidad [Hardin, 1968: 1243].

Pero sería erróneo concluir que el autor desestima la búsqueda de soluciones técnicas para la TC. De hecho, ha insistido en que su formulación describe el trágico resultado como algo exclusivo de los recursos naturales comunes no administrados (no gestionados técnicamente, se entiende) ni por el Estado ni por agentes 
privados. Además de ser empíricamente falsa, como podrá verse, esta afirmación entraña supuestos muy discutibles sobre la naturaleza de toda acción humana, de sus motivaciones, y de la propia noción de ética. Con tales postulados, no sorprende que, según él, la búsqueda de soluciones técnicas para la TC sea inoperante si no hay un cambio en nuestro concepto humanitarista y altruista de ética.

Parte de estos presupuestos se aclaran al reparar en la descripción que, años más tarde, Hardin haría de sus intenciones al formular la Tc. De acuerdo con él, durante más de un siglo los biólogos han faltado a su deber ético y cívico de acercar al público en general la significación humana de la evolución de las especies por selección natural: "La biología abunda en tesis que reclaman un reestructuración masiva de las creencias populares [...] los biólogos deben aceptar la responsabilidad de acercar sus tesis al público [...] 150 años de ausencia de Darwin es más que suficiente” (Hardin, 1986). Hardin se ve a sí mismo embarcado en una cruzada que, suene como suene, comparte bastantes de los planteamientos del darwinismo social del último tercio del siglo XIX. En su opinión, la TC señala las implicaciones éticas de la finitud de la naturaleza concebida como el conjunto de ambientes o nichos ecológicos en los que se desarrolla la lucha inter- e intraespecífica por los recursos, mostrando la fragilidad de ese escenario de escasez ecológica.

Según Hardin, cuando la supervivencia de la especie está en juego, al ser humano no le queda sino regular administrativamente las interacciones individuales en el entorno o ambiente por medio de nociones científicas netamente descriptivas, por ejemplo la capacidad de carga, descrita como "el máximo número de individuos de una especie dada que, teniendo en cuenta cambios de estación y factores como su capacidad de regeneración, un ambiente puede soportar sin comprometer su equilibrio a largo plazo" (Hardin, 1986). La dimensión temporal de este concepto lo convierte en un principio rector de lo que hoy denominaríamos prácticas o actividades sustentables, por lo general definidas como aquellas actividades ambientales destinadas a satisfacer las necesidades de la actual generación sin perjudicar la capacidad de las futuras generaciones para satisfacer sus necesidades. En términos de sustentabilidad, la TC admite ser descrita como una parábola sobre los riesgos de las tendencias al crecimiento acumulativo e indefinido propio del desarrollismo económico y de su gradual uso de materia y energía. La dinámica acumulativa del crecimiento económico indefinido acerca progresivamente los ecosistemas a los umbrales de su capacidad de regeneración (incluyendo su capacidad 
para diluir y eliminar residuos) y, por ende, a los umbrales de su capacidad de carga para sostener las necesidades futuras. Hardin apunta que, frente a la ceguera para la posteridad propia de la tradición ética occidental, una genuina ética ecológica se caracteriza por estar por vez primera orientada hacia el futuro. De ahí que la capacidad de carga haya de figurar irrenunciablemente entre los conceptos de esa ética (Hardin, 2001). Tras la advertencia de la tragedia de los comunes, indica el autor, "el primer mandamiento del nuevo decálogo moral es 'No excederás jamás la capacidad de carga de ningún ambiente” (Hardin, 2001: 53). Es decir, no se debe dejar que viva demasiada gente de manera simultánea si no se quiere destruir la capacidad de carga y por lo tanto reducir cuantitativa o cualitativamente las vidas humanas posibles en periodos subsiguientes. Para obedecer este precepto, la noción de capacidad de carga tendría que administrar las actividades humanas relativas a la reproducción y, en consecuencia, a la alimentación. Como no puede maximizarse a la vez el incremento de población y de calidad de vida (Hardin, 1968: 1244), y apelar a la autocontención reproductiva resulta inútil, la solución de Hardin es la exención del derecho a procrear libremente. Según él, la capacidad de carga es una noción con mayor capacidad normativa que la santidad de la vida, precepto que atribuye a la tradición de las éticas judeocristianas, pero que puede extenderse incluso hasta el budismo.

Como muchos otros pensadores, éste tampoco vacila en apostar por la prevalencia de la capacidad de carga sobre los derechos humanos, ni esconde que tal apuesta depende a su vez de dar por sentada "la legitimidad del deseo humano de maximizar ganancias” (Hardin, 1977). Dicho con otras palabras: para evitar la tragedia, el deseo humano de maximizar ganancias es un derecho (o sea: es un deseo legítimo) que no puede distribuirse entre el total de una población humana. La justicia derivada del concepto ético de capacidad de carga no alcanza para todos.

Hasta aquí, ninguna de las lecturas de Hardin es particularmente novedosa. De hecho, muchas quedaban ya anticipadas en el Primer ensayo sobre población del pastor protestante y economista Robert Malthus (2000), quien veía la tragedia como consecuencia de la asimetría entre el crecimiento demográfico, de orden geométrico, y el incremento de la capacidad productiva de la tierra, de orden aritmético: "Equilibrar el concepto de libertad de procreación con la creencia de que todo el que nace tiene igual derecho sobre los recursos comunes supone encaminar 
al mundo hacia un trágico destino" (Hardin, 1968: 1246). Pero si no todo el mundo puede tener igual derecho ¿qué ética determina quiénes tienen y tendrán derecho, por ejemplo, a recursos ambientales como una atmósfera respirable?

\section{La ética situacionista o del bote salvavidas}

La capacidad de carga como concepto ético nos aboca, dice Hardin, a una ética situacionista o supervivencialista, según la cual la moralidad de una acción no está en función de un imperativo categórico, de un concepto de deber universal, sino del estado del sistema en que tal acción se efectúa. La capacidad de carga de un sistema ecológico, junto con la densidad demográfica de una población de una especie animal no humana dentro de ese ecosistema en una situación temporal determinada, puede hacer aconsejable disminuir o aumentar esa población matando una parte de sus individuos o introduciendo nuevos. Esas prácticas son comunes en el manejo ecológico de recursos naturales y, hoy en día, ningún ambientalista aboga por su erradicación en pro de la santidad de la vida. En términos técnicos, la escasa plasticidad conductual de la mayoría de las especies no humanas facilita determinar sus necesidades, de manera que es posible considerar la calidad de vida como variable independiente y el tamaño de la población como variable dependiente. Pero con las poblaciones de la especie humana las cosas cambian, y mucho. Sin negar su origen biológico, el concepto de necesidad humana es ineludiblemente cultural. La cultura nos "libera” de las necesidades del instinto, ampliando nuestro abanico de respuestas a los estímulos que depara el ambiente, pero al hacerlo la cultura también crea nuevas necesidades y huellas ecológicas. Fijar distributivamente la calidad de vida entre el tamaño de la población humana en un estado temporal del sistema resulta algo tremendamente complejo. Aunque necesario, la capacidad de carga como concepto ético rector de toda conducta humana no parece ser suficiente para evitar en todo caso la TC.

Aparentemente a regañadientes, Hardin admite la exigencia de un concepto de capacidad cultural de carga, recordándonos que "no sólo de pan vive el hombre" (Hardin, 1986). Aun así, y pese a todos sus pronunciamientos éticos, evita con mucho cuidado adentrarse en una ética de las necesidades (en una discusión abierta sobre qué tipo de vida cubre qué necesidades y qué fines de qué generaciones 
futuras de qué seres humanos). Pero sus constantes apelaciones a Malthus dejan claro, además de su antropocentrismo, su radical etnocentrismo:

Malthus entendió perfectamente que la densidad de población debe ser tal que la gente pudiera disfrutar de un filete de carne y una copa de vino para cenar. El concepto malthusiano de capacidad de carga conlleva implícitamente factores culturales. La buena vida, por tanto, debe incluir cierta cantidad razonable (aunque indefinida) de buenos alimentos (verduras frescas, cortes de carne, refrescos y otras bebidas), ropas bonitas y que no se limiten a conservar el calor corporal, hogares cómodos, transporte adecuado, calefacción y aire acondicionado, aparatos electrónicos para el ocio, vacaciones, etc., etc. [Hardin, 1986].

En ese caso, la moralidad del modo de vida occidental (del mismo estilo de vida consumista que muchos, incluyendo el autor de este trabajo, consideran responsable de gran parte de la degradación ambiental) no estaría en función del buen estado del sistema, sino del buen estado de un subsistema privilegiado cuya huella ecológica es tal que amenaza la capacidad de carga de todo el planeta. En esta situación, Hardin tiene claro de quién es la calidad de vida que quiere preservar.

De ahí surge el grito de "sálvese quien pueda" que lanza el pensador con su defensa de la ética del bote salvavidas, metáfora con la que intenta contrarrestar la metáfora de la nave Tierra, dibujada, en su opinión, por una ética de la compasión, tan bien pensante como obsoleta, propia de intelectuales sin coraje que "están menos comprometidos con la verdad que con la estabilidad social, por lo que prefieren el tabú a la confrontación” (Hardin, 1986). Así pues, desafía el precepto de Marx “a cada cual según sus necesidades”: la justicia para todos equivale para él la catástrofe de todos, por lo que en el bote salvavidas no cabemos todos.

Cada nación poderosa puede verse como un bote salvavidas con tripulantes y pasajeros comparativamente ricos. En el mar abierto, nadando entre los botes salvavidas, se hallan los pobres del mundo (unos $2 / 3$ de la población mundial) quienes luchan por un lugar, por modesto que sea, en algún bote salvavidas [...] Nuestra supervivencia es entonces posible dentro del bote sólo si montamos guardia constantemente contra abordajes inoportunos [Hardin, 1974]. 
A los intelectuales del Primer Mundo con mala conciencia, a aquellos que, según él, son simplemente incapaces de soportar con entereza el sufrimiento ajeno, admitiendo que éste es condición necesaria de su propia felicidad, les aconseja intercambiar su lugar por el de algún náufrago, saltando del bote salvavidas.

Los pasos siguientes de su argumentación son predecibles. De hecho, también los había dado ya Malthus en su Primer ensayo sobre población al atacar las leyes de asistencia a los pobres, las Poor Laws promulgadas por utilitaristas y reformistas, los protolaboristas británicos del siglo XIX. Como es sabido, Malthus sostenía que las leyes sociales inglesas destinadas a combatir la pobreza en realidad la fomentan. La asistencia filantrópica a las clases desfavorecidas en términos monetarios causa más miseria de la que palia, al generar un crecimiento demográfico sin producir el correspondiente incremento de la subsistencia o de las existencias disponibles. Malthus llegó a afirmar que estas leyes acaban por crear a los pobres que luego mantienen: "como las provisiones del país deben, como consecuencia del aumento de población, distribuirse en partes más pequeñas para cada uno, resulta evidente que el trabajo de quienes no reciben la ayuda de la beneficencia pública tendrá un poder adquisitivo menor que antes, con lo cual crecerá el número de personas obligadas a recurrir a esta asistencia” (Malthus, 2000: 108). No es la filantropía el medio para luchar contra el dolor ajeno: los vicios y la guerra son, de acuerdo con él, males necesarios para aliviar la infelicidad, puesto que controlan el crecimiento demográfico.

Desde luego, no hay constancia de que Hardin alcanzase esta conclusión públicamente, pero sí de que sugirió que, para evitar estos males demográficos, lo mejor que puede hacerse es, precisamente, japostar contra la cooperación internacional! El proyecto de un Banco Mundial de Alimentos (World Food Bank) representa, en su opinión, un nuevo común disfrazado que conduciría otra vez al desastre ecológico resultante de la TC. Siguiendo la misma idea, autores como Smith (1981) apuntan que la libre empresa administrada de forma privada en competencia en un mercado libre es el mejor antídoto contra la TC. Y, según otros de los actuales seguidores de Hardin, males como el calentamiento global que las políticas ambientales demócratas (como las propuestas por Al Gore) intentan combatir son producto de políticas humanitaristas de administraciones demócratas anteriores. Se trata del bien conocido discurso del individualismo liberal que, apelando exclusivamente al bienestar de los individuos en cuanto puros agentes maximizadores, productores 
y consumidores, simplifica indebidamente las motivaciones de los individuos en cuanto miembros de comunidades de ciudadanos.

\section{Bien común, reciprocidad y ciudadanía}

Muchos críticos de Hardin han negado que sean las acciones locales informadas por conocimientos locales las que típicamente ocasionan ecocidios como la TC. Las destrucciones ecológicas del pasado suelen haber sido causadas por las élites dirigidas por no locales -el caso clásico es la deforestación del litoral del Mediterráneo, producto de siglos de sobreexplotación por la Roma imperial- (Iain Boal en Martinez, 2007). Otros estudios apelan a la autoridad de Engels para defender la existencia de regímenes comunitarios del campesinado en la Europa precapitalista, o al registro antropológico de comunidades indígenas de todo el mundo que vienen regulando durante siglos el uso de sus recursos comunes para impedir la tragedia. De ahí que algunos autores concluyan: "de hecho, no se dio una 'tragedia de los comunes' sino un triunfo: durante cientos de años -y quizá durante miles, aunque carecemos de registros escritos- la tierra fue manejada exitosamente por las comunidades" (Cox, 1985: 60).

Con mucha mayor cautela sobre tal evidencia histórica, la Nobel de Economía (2009) Elinor Ostrom ha seguido una línea parecida, desafiando la conclusión de Hardin, según la cual la TC aboca a la siguiente dicotomía: los recursos naturales comunes deben ser administrados $o$ bien por las autoridades estatales centrales $O$ bien por un sistema de privatización. Con base en una gran variedad de estudios empíricos (algunos de ellos realizados por colaboradores suyos en Latinoamérica) sobre el manejo que los usuarios comunitarios hacen de bosques, minerales, alimentos, zonas turísticas, bancos de peces, pastos comunales, ríos, lagos y aguas subterráneas, Ostrom concluye que en muchos casos el trágico pronóstico de Hardin no se ha cumplido. Con todo, su análisis nos previene contra el ansia de generalidad: en muchos otros casos, el manejo comunitario de los recursos naturales sí ha conducido a la catástrofe (Weber, Garcia-Marmolejo y Reyna-Hurtado, 2006). Inevitablemente, Ostrom llega a la misma conclusión que otros autores: la Tc puede producirse o no con independencia del sistema de administración o la estructura de la propiedad del recurso: "la investigación no ha encontrado 'curas seguras' para solucionar los problemas complejos de gobernanza de un bien común. El fracaso 
aparece en relación con la propiedad privada, la gubernamental y la común” (Ostrom, 2000). Algo más que el régimen de propiedad debe ser tenido en cuenta a la hora de explicar, predecir y prevenir la TC.

No es posible analizar aquí todos los pormenores de la investigación empírica de Ostrom en torno al conjunto de factores culturales (tecnológicos, políticos y, sobre todo, institucionales) que condicionan la sustentabilidad (o, alternativamente, el colapso) en el manejo comunitario de los recursos naturales. Empero, sí lo es señalar las implicaciones de algunos de sus resultados experimentales, ciertamente pertinentes para dejar algún lugar, por modesto que sea, a la ética ambiental entre las disciplinas ambientales. Según Ostrom, estos resultados manifiestan que las comunidades en las que hay condiciones de comunicación, complementariedad de expectativas y reconocimiento mutuo (de manera que el agente, "si la situación se repite [...] puede usar la reciprocidad para ganarse la reputación de ser confiable” —Ostrom, 2000—) son más robustas para evitar la TC. "Los valores individuales en sí no son suficientes para solucionar los problemas de esta índole. Sin instituciones que faciliten la construcción de reciprocidad, confianza y honradez, los ciudadanos enfrentan un reto real" (Ostrom, 2000). La Nobel y sus colaboradores aportan numerosos estudios de caso de comunidades que han mostrado resultados óptimos en el manejo sustentable de los recursos comunes (Ostrom et al., 1999). Gusten o no, sus análisis indican que, si bien por sí mismos no son suficientes, algunos valores de carácter ético como la reciprocidad y la confianza son condiciones necesarias para enfrentarse a la TC. El reconocimiento de los demás, la obtención de un valor como agente digno de respeto y confianza por su aportación a una comunidad que se sabe interdependiente, opera de hecho como contrapeso a la expectativa de incrementar beneficios individuales ocurra lo que ocurra, la cual conduce a la TC. El análisis de Ostrom, como con anterioridad el de Axelrod y Hamilton (1981), expone decisivamente que tales valores son importantes para generar una racionalidad colectiva con base en la cooperación que resulte ser sustentable y evolutivamente adaptativa. Dicho de otro modo, la racionalidad ambiental en el manejo de recursos naturales no puede limitarse a lo que Max Weber llamaba racionalidad formal o instrumental en cuanto productora de vínculos operacionales entre medios y fines, cuya eficiencia puede ser determinada de manera cuantitativa (Weber, 1981: 64).

Ahora bien, las consecuencias que para la racionalidad ambiental tendrían los resultados que Ostrom extrae de sus diseños experimentales de la TC sólo pueden 
interpretarse desde la perspectiva de la complementariedad entre racionalidad instrumental y axiológica. Que los efectos de la TC puedan minimizarse en aquellas comunidades donde la información fluye de forma más equitativa y los lazos intersubjetivos son más estrechos no significa que sea factible resolver conflictos ambientales simplemente con el cultivo de los valores de la comunicación y la comunalidad, pues en la génesis y el desarrollo de esos valores también intervienen relaciones de eficiencia entre medios o recursos y fines. Además, se determine numéricamente o no, el concepto de eficiencia de la racionalidad instrumental es intrínsecamente económico, al menos bajo la aceptable definición de economía como "la ciencia que estudia el comportamiento humano en cuanto relación entre fines y medios escasos que tienen usos alternativos" (Robbins, 1932: 16). Pero la cuestión fundamental de la racionalidad no es sólo la elección (choice) entre usos alternativos de medios escasos, sino también entre fines y metas racionalmente aceptables de acuerdo con los medios disponibles (Weber, 1981: 47). Según esta interpretación, los resultados de Ostrom evidencian que los miembros de las comunidades donde se logró minimizar la TC realizaron esa elección contando con valores de reciprocidad que son instrumentales para la cooperación ciudadana y por consiguiente para la supervivencia de una comunidad enteramente dependiente de su entorno (en el ejemplo de Ostrom, la reciprocidad, la confianza y la honradez). Cuando, siguiendo las pistas de Ostrom, se equipara la TC con los problemas contemporáneos derivados de la huella ecológica y la sustentabilidad de nuestros modos de vida, considerando la atmósfera o los mares como un bien global común, por ejemplo, resulta legítimo concluir que al menos ciertos valores éticos como la reciprocidad deben integrarse en nuestra racionalidad, toda vez que aspiremos a enfrentarnos científicamente, con la fuerza de las razones, a problemas tales como el cambio climático o la escasez de recursos hídricos y pesqueros. La racionalidad ambiental no puede excluir una racionalidad instrumental para la cual la eficiencia en la relación entre medios y fines debe ser expresada en términos de un cálculo con unidades cuantificables de cambio entre diferentes servicios ambientales. Pero por muy escurridizos que sean, o por muy molestos en términos de cuantificación o determinación numérica de su efectividad en el manejo sustentable de recursos comunes, los factores axiológicos no pueden ser excluidos de la racionalidad ambiental.

Para seguir explorando el difícil terreno axiológico de la racionalidad ambiental, es necesario descartar la tentación totalitaria de aplicar de cabo a rabo 
soluciones puramente técnicas mediante la anulación por la fuerza de los efectos ambientalmente destructivos de la conducta individual humana. Ninguna historia honesta del pensamiento ambiental puede dejar de recordar que fue probablemente el régimen nacionalsocialista, durante los años treinta del pasado siglo, el que promulgó una de las primeras legislaciones ambientales sistemáticas y de gran alcance. Independientemente de los valores "patrióticos" de suelo, sangre, raza y naturaleza aria, empleados como propaganda para la justificación popular de sus leyes ecológicas de 1933, 1934 y 1935 (Ferry, 1992), el autoritarismo ecológico fue legitimado por pensadores como Martin Heidegger, quien vaticinaba que la democracia pluralista y el humanismo eran la peor manera de hacer frente a las consecuencias globales o planetarias de la tecnología de occidente (Heidegger, 1976). En idéntica tesitura, James Lovelock, biólogo creador de la hipótesis de Gaia, que hace de la Tierra un único organismo autónomo, llamaba a una suspensión autoritaria de las libertades civiles democráticas ante las inminentes situaciones de emergencia ocasionadas por el cambio climático (James Lovelock en Hickman, 2010). La idea de un gobierno de expertos, a quienes su presunto conocimiento de la verdad legitima para imponer autoritariamente soluciones técnicas para el bien común, ha hechizado a los seres humanos por lo menos desde Platón (y ha despertado su suspicacia por lo menos desde Aristóteles). Pero por muchos problemas que pretenda resolver, la administración tecnocrática encuentra otras dificultades que reintroducen cuestiones axiológicas como la reciprocidad y la ciudadanía. Hardin reconoce implícitamente este extremo al admitir el problema de "Quis custodiet ipsos custodes" como algo propio de toda ética situacionista en la medida en que intente hacer frente a la TC.

Si la moralidad de una acción, como defiende la ética situacionista, está en función del estado del sistema, las leyes que norman las interacciones dentro de éste dejan de ser universalmente obligatorias, pues es imposible establecer de antemano cuáles serán los futuros estados del sistema. De acuerdo con Hardin, esa incertidumbre objetiva exige leyes que doten al administrador, estatutariamente, del poder para llevar a cabo decisiones instantáneas y específicas dentro de un marco jurídico general, teniendo en cuenta interpretaciones expertas del estado del sistema. Pero expertos y administradores son humanos y, por lo tanto, falibles. Sus decisiones pueden responder a su propio interés. ¿Y quién juzga sus decisiones? ¿Quién vigila al vigilante? 
Puesto que resulta prácticamente imposible mencionar todas las condiciones bajo las cuales es seguro quemar basura en el patio trasero o manejar un coche sin control anticontaminante, con las leyes delegamos los detalles a las oficinas. El resultado es una ley administrativa, la cual es lógicamente temida por la vieja razón - ¿Quién ha de vigilar a los propios vigilantes?-. Los administradores, al tratar de evaluar la moralidad de los actos en la totalidad del sistema, están singularmente expuestos a la corrupción, generando un gobierno de hombres y no de leyes. La prohibición es fácil de legislar (pero no necesariamente fácil de imponer); pero ¿cómo legislar la moderación? La experiencia indica que ésta puede ser alcanzada mejor a través de la acción de la ley administrativa. Limitamos innecesariamente las posibilidades si suponemos que los sentimientos de Quis custodiet nos niegan el uso de la ley administrativa. Deberíamos mejor tener la frase como un perpetuo recordatorio de temibles peligros que no podemos evitar [Hardin, 1968: 1245].

Hardin admite así la imposibilidad de eliminar el problema de la corrupción administrativa de los propios administradores de los recursos ambientales comunes, pero parece aconsejar que se inviertan los términos y se tolere como un mal menor frente a la Tc: se diría que, como la injusticia, la corrupción es preferible a la ruina de todos. "La necesidad ecológica puede presionarnos a abandonar el ideal de un gobierno de leyes y no de hombres” (Hardin, 1977).

Qué tan menor sea ese mal, o qué tanto se haya generalizado la corrupción hasta el punto de provocar precisamente la ruina de todos, es algo sujeto a discusión. La experiencia de las últimas décadas (incluyendo como síntoma la proliferación de códigos éticos para funcionarios públicos en todos los países) no permite hacer un balance tan condescendiente como el de Hardin. La corrupción administrativa ha demostrado fehacientemente ser también origen, y no sólo resultado, de catástrofes ambientales. Desde luego, debe romperse la ecuación entre autoridad e impunidad con leyes adecuadas, pero toda administración ambiental implica cierto grado de discrecionalidad que ninguna técnica puede eliminar. Las consecuencias son más graves cuando se trata de las acciones de los vigilantes, pero ese grado de discrecionalidad se aplica a las del conjunto de la población. Más allá de nuestra simpatía o antipatía por la fórmula de vigilar y castigar, la propia naturaleza de la mayoría de los bienes ambientales comunes desaconseja el incremento indefinido de 
leyes administrativas si se desatiende la formación ciudadana en la reciprocidad de derechos y deberes. Estudios como los de Ostrom hacen posible estimar el valor ambiental de la consideración recíproca obtenida por los ciudadanos al actuar como ciudadanos ante situaciones problemáticas o conflictos ambientales. En términos ambientales, la moderación que exige la necesidad ecológica (Hardin, 1977) y el reconocimiento ciudadano de derechos y deberes son las dos caras de la misma moneda. La racionalidad ambiental para el uso sustentable de los bienes comunes no puede prescindir de una ética de la reciprocidad ciudadana.

\section{El cambio climático como tragedia de los comunes}

Algunos problemas ambientales contemporáneos pueden ser adecuadamente reformulados desde esta visión. La estabilidad climática depende en parte de un bien común como la atmósfera, la cual a su vez facilita una serie de servicios ambientales, por ejemplo aire puro, un régimen pluvial adecuado para nuestros cultivos, condiciones generales de salubridad, trabajo y ocio, entre otros. En este sentido, la estabilidad climática, como la salud, los caminos y los puentes o la iluminación pública, son bienes comunes, esto es, servicios a los que tienen acceso todos los miembros de determinado grupo social independientemente de que hayan contribuido o no a su producción o conservación. En nuestro caso, independientemente de que una persona haya frenado o no su emisión de gases de efecto invernadero, disfrutará de los beneficios de un clima estable: no podemos hacer que quienes más contaminan respiren menos aire puro, o que dejen de gozar de temperaturas moderadas o sean más perjudicados por los huracanes. Además, aunque para que exista el bien común no es necesario que todos y cada uno de los miembros de un grupo social realicen su aportación, sí ha de haber un número suficiente de individuos que contribuyan (ahorrando más energía eléctrica, usando menos vasos de plástico, comiendo menos carne o separando su basura, por ejemplo). En términos puramente instrumentales, al individuo que contribuye su aportación le reporta a corto plazo más costos que beneficios: quizá tenga que privarse de alguna comodidad por ahorrar luz o usar menos bolsas de plástico, o tenga que invertir tiempo y dinero al separar su basura o al reparar su impresora en vez de comprar una nueva. Pero, como en el caso de los impuestos para bienes comunes como la seguridad pública o el alumbrado, el individuo que contribuye prefiere el servicio 
que obtendrá finalmente a cambio de su aportación al bien público que cualquier otro servicio del que disfrutaría con el monto de su cooperación (prefiere el aire puro o la ausencia de inundaciones que cualquier otra cosa que pudiera obtener con los costos que acarrea separar su basura o lavar su taza en vez de utilizar un recipiente desechable para su café). Todos estos rasgos del bien común sitúan la racionalidad instrumental del individuo o contribuyente en la siguiente coyuntura: "Si ya hay masa crítica para obtener el bien, puedo ahorrarme el costo de la cooperación; si no la hay, me perjudica contribuir. Así que mejor no coopero”. Pero si todo el mundo se comporta como un aprovechado (free-rider) o se abstiene de cooperar, algo muy parecido al resultado de la TC es inevitable, y luchar contra el calentamiento global, por ejemplo, sería un esfuerzo inútil. La tragedia del cambio climático demuestra ser inevitable si y sólo si la racionalidad instrumental y la racionalidad ambiental son coextensivas. Sin embargo, como ha podido observarse, no siempre es así.

En algunos contextos y situaciones, la retribución que la conducta comporta en términos de reconocimiento público parece ser motivo suficiente para la acción cooperativa. En otros, no. Todo lo contrario: en ellos, lo que nos garantiza nuestra cooperación es la reputación de estúpidos o tontos. En caso de obtener el bien común, estúpidos por haber contribuido cuando pudimos haberlo conseguido gratis; en caso de no obtenerlo, pueden considerarnos doblemente estúpidos o losers: perdemos tanto por haber contribuido como por no haberlo conseguido. No es casual que esos contextos de no cooperación sean habitualmente de corrupción generalizada. Pero de algo sí es posible estar seguros: las comunidades que propician estos últimos contextos, reforzando negativamente la cooperación y menguando el prestigio o reconocimiento público, parecen fatalmente dirigidas a catástrofes ambientales tipo la TC. No parece muy prudente centrarse de manera exclusiva en aspectos técnicos e instrumentales de la racionalidad ambiental y prescindir de los aspectos éticos de nuestras acciones, como su carácter universalizable o el respeto y el deber de los ciudadanos con los ciudadanos. Al fin y al cabo, si algo indican los estudios sobre la evolución de la cooperación en bienes comunes es que la libre comunicación, la reciprocidad, la confianza y la honestidad han estado presentes en muchas de las comunidades que han logrado, cuando menos, minimizar la degradación ambiental que la TC conlleva. 


\section{Conclusiones}

El presente trabajo ha intentado mostrar que la propia formulabilidad de la TC presupone la validez de la teoría instrumental de la racionalidad, según la cual el agente racional orienta sus acciones únicamente a la maximización de beneficios. Se apuntó cómo, en consonancia con sus supuestos malthusianos, Garrett Hardin extrae un concepto ético de capacidad de carga que entra en grave en conflicto con las tesis de la democracia social y la justicia distributiva, resumido en una ética situacionista, supervivencialista o del bote salvavidas. La defensa de la justicia social distributiva exige pues introducir nociones axiológicas en la descripción de la acción racional sin desatender los límites ecológicos que la noción de capacidad de carga impone a partir de la TC. Este artículo apela a los estudios empíricos de la economista Elinor Ostrom, quien demuestra que las nociones éticas de confianza, reciprocidad, honradez y reconocimiento público o prestigio desempeñan un papel importante en las acciones racionales de los individuos pertenecientes a comunidades que han logrado al menos minimizar los efectos de la Tc. Esas nociones integran una ética de la ciudadanía que resulta igualmente necesaria para una aproximación constructiva a los problemas del cambio climático, entendidos como una subclase de los problemas "sin solución exclusivamente técnica" que la tragedia de los comunes representa.

\section{AgRAdecimientos}

El autor del presente trabajo quiere agradecer al maestro Roberto Acosta y al doctor José Hernández su apoyo para realizar este y otros artículos en las excelentes condiciones de trabajo que brinda la División de Ciencias e Ingenierías de la Universidad de Quintana Roo, y también a los alumnos de la primera generación de la Maestría en Educación Ambiental su estimulante discusión de la obra de Garrett Hardin. 


\section{FUENTES CONSULTADAS}

Axelrod, R. y W. Hamilton (1981). "The Evolution of Cooperation”. Science, 211, 1390-1396.

Cox, S. (1985). "No Tragedy on the Commons". Environmental Ethics, 7, 50-73.

Ferry, L. (1992). The New Ecological Order. Chicago: Chicago University Press.

Hardin, G. (1968). "The Tragedy of the Commons”. Science, 162, 1243-1248.

(1974). Lifeboat Ethics: the Case Against Helping the Poor [en línea]. Disponible en: http://www.garretthardinsociety.org/articles/art_lifeboat_ ethics_case_against_helping_poor.html [2011, 11 de mayo].

(1977). Ethical Implications of Carrying Capacity [en línea]. Disponible en: http://www.garretthardinsociety.org/articles/art_ethical_implications. html [2011, 20 de agosto].

(1986). Cultural Carrying Capacity [en línea]. Disponible en: http://www. garretthardinsociety.org/articles/art_cultural_carrying_capacity.html [2011, 6 de mayo].

- (2001). "Carrying Capacity as an Ethical Concept". The Social Contract, otoño de 2001, 48-57.

Heidegger, M. (1976). “Nur noch ein Gott Kann uns retten”. Der Spiegel, 23, 31 de mayo de 1976, 193-219.

Heilbroner, R. (1980). An Inquiry Into the Human Prospect. Nueva York: W. W. Norton.

Hickman, L. (2010). "James Lovelock: Humans are too Stupid to Prevent Climate Change”. The Guardian, 29 de marzo de 2010 [en línea]. Disponible en: http://www.guardian.co.uk/science/2010/mar/29/james-lovelock-climatechange [2010, 4 de abril].

Malthus, R. (2000). Primer ensayo sobre población. Madrid: Alianza Editorial.

Martinez, D. (2007). "Iain Boal in conversation with David Martinez. Specters of Malthus: Scarcity, Poverty, Apocalypse". Counter Punch, 11 de septiembre de 2007 [en línea]. Disponible en: http://www.counterpunch. org/2007/09/11/specters-of-malthus-scarcity-poverty-apocalypse/ [2011, 29 de junio]. 
Ostrom, E. (2000). El gobierno de los bienes comunes desde el punto de vista de la ciudadanía [en línea]. Disponible en: http://www.colsan.edu.mx/ investigacion/aguaysociedad/proyectogro2/Biblioteca/Bibliografia/ M \% F3dulo \% 205/EL \% 20GOBIERNO \% 20DE \% 20LOS \% 20BIENES \% 20 COMUNES \% 20DESDE \% 20EL \% 20PUNTO \% 20DE \% 20VISTA \% 20DE \% 20 LA\%20CIUDADAN\%CDA.pdf [2011, 27 de julio].

Ostrom, E., et al. (1999). "Revisiting the Commons: Local Lessons, Global Challenges”. Science, 284, 278-282.

Robbins, L. (1932). An Essay on the Nature and Significance of Economic Science. Londres: Macmillan.

Smith, R. (1981). "Resolving the Tragedy of the Commons by Creating Private Property Rights in Wildlife”. The Cato Journal, 1 (2), otoño de 1981, 439469.

Weber, M. (1981). Economía y sociedad. México: Fondo de Cultura Económica.

Weber, M., G. Garcia-Marmolejo y R. Reyna-Hurtado (2006). "The Tragedy of the Commons: Wildlife Management in Southern Mexico". Wildlife Society Bulletin, 34 (5), 1480-1488. 